\title{
MDT approach to improve attendance of outpatient appointments and scans for geriatric inpatients
}

\author{
Author: Raees Lunat ${ }^{\mathrm{A}}$
}

While working on a geriatric ward, I noticed many of the patients were not responsible for their own care and that often it was done by family/community teams. Thus, when they came into hospital, they were often missing planned outpatient appointments/ investigations which were scheduled for them. This led to poor patient care and increased missed appointments. Imperatively many of these patients were vulnerable and it would improve their quality of life greatly to have all investigations while an inpatient rather than coming back repeatedly for single appointments

I was able to assess the extent of this issue by discussing with our IT team, they ran a model which displayed all patients on geriatric wards who had upcoming outpatient appointments/investigations. I then discussed with local teams on wards and decided that doctors, head nurses and ward clerks would be notified twice weekly about upcoming appointments. My analysis was based on looking at how many of those who had appointments attended them and, if they did not, why not.

My research directly tackled the initial challenge as it has allowed elderly inpatients to get to previously scheduled appointments, they have benefited from reviews which they were meant to get and are not having duplicate imaging/appointments. The benefits include improved patient care, improved outpatient attendance, lower return to hospital for appointments and fewer missed appointments. The main problems included getting the various multidisciplinary team members to fully participate in the project and incorporate it into their daily board round.

The results identified the various differing appointment types and ensured that the majority of patients attended these. The result of these outcomes was that when the new electronic system came into play at the hospital, all outpatient appointments were made visible to the accessing healthcare professional, hence ensuring that all inpatients had the opportunity to attend upcoming appointments.

\section{Conflicts of interest}

None declared. 